\title{
Pif1 overcomes a quadruplex hurdle
}

G-quadruplex (G4) structures, formed by non-canonical intramolecular $\mathrm{G} \bullet \mathrm{G}$ base pairs, are predicted to be prevalent in the genome. Their stable nature suggests that they might pose problems for DNA in vivo and, indeed, Zakian and colleagues have found that, in Saccharomyces cerevisiae, G4

given that Pif1 associates with only a subset of G4 motifs, it is likely that it shares this responsibility with other helicases structures slow down replication forks and that this is counteracted by the Pif1 helicase.

Pifl stands out among the helicases that can resolve G4 structures in vitro for its potent unwinding activity. In vivo, Pif1 is known to have several roles, including telomere maintenance and DNA replication. So, Zakian and colleagues set out to address whether the ability of Pif1 to unwind G4 structures might be relevant to these functions in vivo. Using chromatin immunoprecipitation,

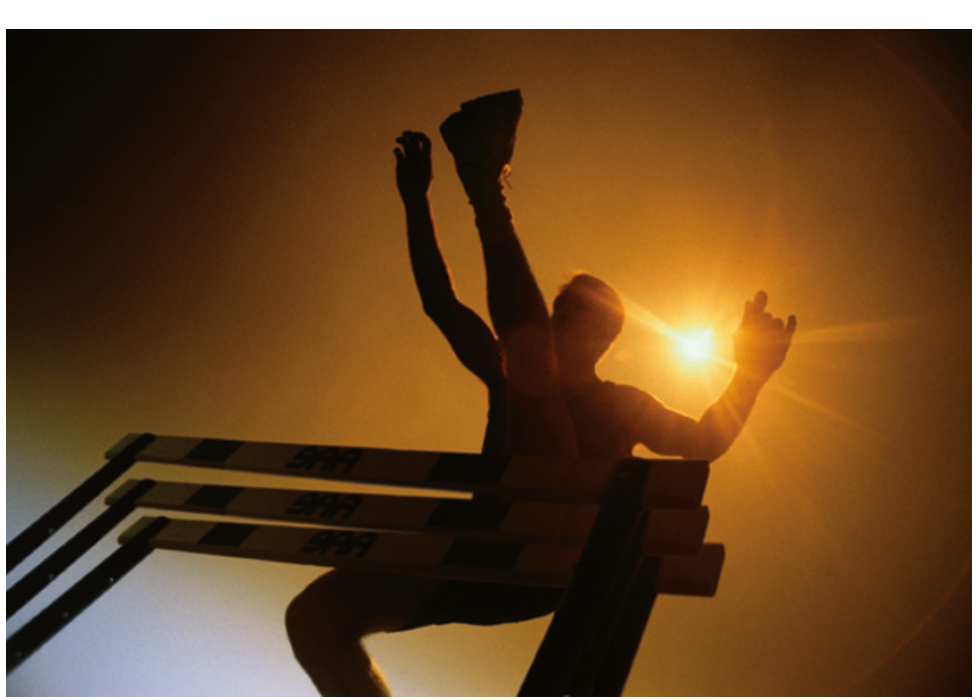

in Pif1-deficient cells, and this correlated with the formation of DNA double-stranded breaks. So, one possibility is that the pausing of replication forks that occurs when $\mathrm{G} 4$ structures accumulate in the absence of Pif1 promotes DNA breakage and subsequent recombination events.

If G4 motifs do disrupt the normal progression of replication forks and increase DNA breaks, their accumulation might be expected to reduce cell growth when cells experience replication stress. The authors tested this by increasing the copy number of G4 motifs and then examining what happens when cells experience replication stress in hydroxyurea. They found that this stress led to reduced cell growth in Pif1-mutant cells and also correlated with spontaneous mutation at the G4 motifs and subsequent rescue of replication progression and DNA breakage.

The authors propose that a key role of Pif1 is to ensure that G4 structures are resolved so that DNA replication can progress normally. However, given that Pif1 associates with only a subset of G4 motifs, it is likely that it shares this responsibility with other helicases. It will therefore be important to see how these may act in concert to overcome such structural obstacles in the genome. Alison Schuldt

ORIGINAL RESEARCH PAPER Paeschke, K., Capra, J. A. \& Zakian, V. A. DNA replication through G-quadruplex motifs is promoted by the Saccharomyces cerevisiae Pif1 DNA helicase. Cell 145, 678-691 (2011) 